of the equations used in the physical studies of chainlength, distribution and the kinetics of polymerization are set out in most cases as a mathematical exercise. It might well be that this could have formed a more mathematical monograph on these known aspects of polymer structure. In this case the derivation of the formulæ could have been curtailed in the present work, and the physical significance of the results to the understanding of polymer structure given greater emphasis. It should, however, be pointed out that the references in this field are good. The chapter on molecular weight determination has two pages devoted to chemical methods and about twenty to a detailed description of physical methods.

Chapter 6, Chapter 10 and Chapter 11 give compara. tively simple descriptions of cellulose, proteins (mainly wool) and silk fibres respectively. Other chapters then deal with the physics and chemistry of these materials, and this treatment of the subjects shows wide variations in levels of presentation. This is particularly so on p. 194 et seq., where an advanced treatment of the reactions of the hydroxyl groups in cellulose is given. I feel that, in this work, accessibility of the glucose residue with special reference to erystalline packing should be considered in any theory applicable to cellulose fibres. Chapter 9 describes the work which has been done on synthetic polypeptides, but for students it might have been a help to correlate the natural and synthetic $\alpha$ - and $\beta$-structures even if the treatment had been critical of some of the theories advanced.

Chapter 12 is devoted to the crystalline structure of polymers, and there is no doubt that a re-editing of this chapter should be done. It must be pointed out that many of the X-ray diagrams are incorrectly aligned (this can be readily corrected by an arrow giving the fibre axis) and the text of pp. 321 et seq. has been confused. Fig. $77 a$ is wrongly oriented but correctly labelled, Fig. $77 b$ is not ramie but most likely polyvinyl alcohol or a similar vinyl fibre. Figs. $77 c$ and $77 d$ are incorrectly oriented. Fig. $77 e$ is most likely a polyacrylic fibre with a $\mathrm{TiO}_{2}$ dilustrant included, and Fig. $77 f$ is an incorrectly oriented picture of ramie taken on a circular camera and contains no $\mathrm{TiO}_{2}$.

Chapters 13 and 14 are good generally, the former on melting and crystallization of man-made polymers, and the latter on accessibility and crystallinity. The chapters on fibre extrusion should probably have included the equations of extrusion. This would then have clarified the statement on p. 432, which suggests that yarn diameter (presumably denier) on extrusion is a function of the diameter of the jet holes. Probably the main criticism of this work is that Prof. Peters has attempted to cover too wide a field and in doing so has, maybe of necessity, had to vary the standard of treatment of his material. The scope, however, is good, and there is nothing in the book that could not be rectified by a good re-editing.

F. HAPPEY

\section{QUANTITATIVE ORGANIC ANALYSIS}

Quantitative Organic Analysis via Functional Groups By Dr. Sidney Siggia. Third edition. Pp. xii +697 . (New York and London: John Wiley and Sons, Inc., 1963.) I45s.

7

HE third edition of Dr. Siggia's book is described on the dust-jacket as being about double the size of the previous edition. The price of the book has been almost quadrupled. Neither increase would have been entirely necessary had the author been a little more selective in his choice of material. The book consists largely of reprints or partial reprints of papers and articles from other books and journals. The sources are quite clearly indicated and the extracts are variously described as being, reprinted with permission, reprinted in part, reprinted, adapted or adopted or are simply referred to. Some of the papers are Dr. Siggia's own, but his main role has been that of a collector and he has drawn heavily on the American Chemical Society publication, Analytical Chemistry. Simple reference to the original journals would have sufficed for much of the original discussion, results, tables and diagrams. Only the essential analytical procedure need have been retained in full. Applied throughout the book this would have reduced the size, lowered the price and in all probability increased the sales.

The book is so planned as to devote one chapter to the methods of analysis for each functional group considered. These include hydroxyl and carbonyl groups, carboxylic acids and derivatives, alkoxyl and epoxide groups, peroxides, unsaturation, acetate, ketals, amino and imino groups, hydrazides, diazonium salts, quaternary ammonium compounds, isocyanates and isothiocyanates, sulphides, sulphoxides and sulphonic acids and derivatives.

Each section is preceded by a discussion of the particular chemical reactions involved in the determination and this is followed by the methods derived from them. Methods are laid out in standard fashion indicating apparatus and reagent requirements followed by procedure and calculation. All the methods chosen have been tried in the author's laboratory.

At the end of the book there are chapters dealing with the development of new analytical methods, the role of quantitative functional group determination in the identification of organic compounds, the use of differential rate of reaction to analyse mixtures and the weighing of volatile and corrosive liquids. The chapter on the role of quantitative functional analysis might well have come first rather than almost last, while that on woighing corrosive liquids, although useful, would have been better had it been enlarged to deal with sampling and sample-handling techniques in general. The analyses of mixtures by differential rate of reaction is illustrated by reference to mixtures of alcohols, amines, esters, etc., good results being obtained in some instances.

The section on physical methods of analysis has been excluded from this edition. In view of the importance of these methods in modern organic analysis, not only should it have been retained, but also it should have been enlarged.

This book is not likely to recommend itself to students because of its high cost. It may be useful, however, to those laboratories engaged in general quantitative organic chemistry where library facilities are limited.

\section{P. J. COOPER}

\section{OXO-COMPOUNDS}

Inorganic Adduct Molecules of Oxo-Compounds By Ingvar Lindquist. (Anorgansiche und Allgemeine Chemie in Einzeldarstellungen, Band 4.) Pp. vi +129. (Berlin: Springer-Verlag, 1963.) 20 D.M.

A $T$ first sight this book appears to be something midway between a review article and a section of Gmelin. Resemblance to the latter owes much to the style of printing, which makes for extremely tedious reading.

The book is concerned with adducts formed by Lewis bases in which the donor atom is oxygen, but it is limited to a discussion of so-called 'oxo' donors in which the oxygen atom is linked to only one other atom. These donors include molecules containing the following groupings:

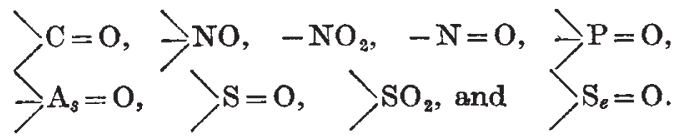

Donors such as ethers are not included, however. 